\title{
In Vivo Two-Photon Imaging of Dendritic Spines in Marmoset Neocortex ${ }^{1,2,3}$
}

\author{
Osamu Sadakane, ${ }^{1,2}$ Akiya Watakabe, ${ }^{1,2}$ Masanari Ohtsuka, ${ }^{1,2}$ Masafumi Takaji, ${ }^{1,2}$ Tetsuya Sasaki, ${ }^{3}$ \\ Masatoshi Kasai, ${ }^{4}$ Tadashi Isa, ${ }^{4}$ Go Kato, ${ }^{5}$ DU Junichi Nabekura, ${ }^{5}$ Hiroaki Mizukami, ${ }^{6}$ Keiya Ozawa, ${ }^{6}$ \\ Hiroshi Kawasaki, ${ }^{7}$ and ${ }^{1}$ Tetsuo Yamamori ${ }^{1,2}$
}

\section{DOI:http://dx.doi.org/10.1523/ENEURO.0019-15.2015}

\begin{abstract}
${ }^{1}$ Laboratory for Molecular Analysis of Higher Brain Function, Brain Science Institute, RIKEN, Wako, Saitama 351-0198, Japan, ${ }^{2}$ Division of Brain Biology, National Institute for Basic Biology, Aichi 444-8585, Japan, ${ }^{3}$ Department of Ultrastructural Research, National Center of Neurology and Psychiatry, National Institute of Neuroscience, Tokyo 187-8502, Japan, ${ }^{4}$ Division of Behavioral Development, National Institute for Physiological Science, Aichi 444-8585, Japan, ${ }^{5}$ Division of Homeostatic Development, National Institute for Physiological Science, Aichi 444-8585, Japan, ${ }^{6}$ Division of Genetic Therapeutics, Center for Molecular Medicine, Jichi Medical University, Tochigi 329-0498, Japan, ${ }^{7}$ Department of Biophysical Genetics, Graduate School of Medical Sciences, Kanazawa University, Ishikawa 920-8640, Japan
\end{abstract}

\begin{abstract}
Two-photon microscopy in combination with a technique involving the artificial expression of fluorescent protein has enabled the direct observation of dendritic spines in living brains. However, the application of this method to primate brains has been hindered by the lack of appropriate labeling techniques for visualizing dendritic spines. Here, we developed an adeno-associated virus vector-based fluorescent protein expression system for visualizing dendritic spines in vivo in the marmoset neocortex. For the clear visualization of each spine, the expression of reporter fluorescent protein should be both sparse and strong. To fulfill these requirements, we amplified fluorescent signals using the tetracycline transactivator (tTA)-tetracycline-responsive element system and by titrating down the amount of Thy1S promoter-driven tTA for sparse expression. By this method, we were able to visualize dendritic spines in the marmoset cortex by two-photon microscopy in vivo and analyze the turnover of spines in the prefrontal cortex. Our results demonstrated that short spines in the marmoset cortex tend to change more frequently than long spines. The comparison of in vivo samples with fixed samples showed that we did not detect all existing spines by our method. Although we found glial cell proliferation, the damage of tissues caused by window construction was relatively small, judging from the comparison of spine length between samples with or without window construction. Our new labeling technique for two-photon imaging to visualize in vivo dendritic spines of the marmoset neocortex can be applicable to examining circuit reorganization and synaptic plasticity in primates.
\end{abstract}

Key words: dendrite; marmoset; neocortex; primate; spine

\section{Significance Statement}

Investigation of nonhuman primate brains is important for the understanding of the human brain. However, because of technical difficulties, several important methods that have been used in rodent studies are not available for primate studies. Two-photon imaging of dendritic spines has been used in rodent studies, which clarified the basis of neural circuit plasticity, but there has been no report of the application of this imaging method to primate brains. Therefore, in this study, we developed an adeno-associated virus vector-based fluorescent protein expression system for use in the studies of the marmoset neocortex. Our approach enabled the sparse yet strong expression of fluorescent protein in neurons. This labeling technique will be applicable to research into the circuit reorganization of primate brains. 


\section{Introduction}

Direct observation of fine neuronal morphologies such as dendritic spines in living brains has been made possible with techniques involving the expression of fluorescent protein in neurons of living animals in combination with two-photon microscopy. Many researchers prefer to use transgenic mouse lines such as Thy1-GFP and Thy1YFP mice for their imaging studies of dendritic spines, because of the stable and strong expression of fluorescent protein in a subpopulation of neurons in these mouse lines (Feng et al., 2000; Grutzendler et al., 2002; Trachtenberg et al., 2002; Holtmaat et al., 2005; Zuo et al., 2005; Kim and Nabekura, 2011; Fu et al., 2012). The advancement of two-photon microscopy was also a key factor for the application of spine-imaging techniques to living animals (Denk et al., 1990; Denk and Svoboda, 1997). The long-wavelength light used in two-photon microscopy penetrates a specimen with less scattering than the shortwavelength light used in other conventional microscopy techniques such as confocal microscopy; thus, it is possible to observe signals from deep regions in a certain thick tissue.

Dendritic spine imaging by two-photon microscopy has been almost exclusively performed on rodent brains, and there have been only a few studies in which the brains of larger species such as primates and carnivores have been examined. One major reason for the lack of studies of such animals with larger brains is that there has been no standard method for in vivo imaging in these species. Transgenic monkey studies have been generated (Sasaki et al., 2009; Niu et al., 2010), but no primate model that strongly expresses fluorescent protein for observing signals in vivo has been established. Spine imaging of the primary visual cortex of ferrets using an expression system with a virus vector was reported (Yu et al., 2011). In the case of primates, one group studied the neuronal morphology in the primary visual cortex of macaque mon-

Received February 27, 2015; accepted July 27, 2015; First published August 27, 2015.

${ }^{1}$ The authors declare no competing financial interests.

${ }^{2}$ Author contributions: O.S., A.W., T.I., J.N., K.O., H.K., and T.Y. designed research; O.S., A.W., M.O., M.T., T.S., M.K., G.K., and H.M. performed research; O.S. analyzed data; O.S., A.W., M.T., and T.Y. wrote the paper.

${ }^{3}$ This research is supported by the Strategic Research Program for Brain Sciences of the Ministry of Education, Culture, Sports, Science and Technology, MEXT, Japan (to T.Y., T.I., and K.O.); by the program for Brain Mapping by Integrated Neurotechnologies for Disease Studies (Brain/MINDS) from Ministry of Education, Culture, Sports Science, MEXT, and the Japan Agency for Medical Research and Development, AMED (to T.Y.); and by the NIBB Collaborative Research Program (13-308)(to T.S.)

Acknowledgements: Confocal images were acquired at the Spectrography and Bioimaging Facility, National Institute for Basic Biology Core Research Facilities.

Correspondence should be addressed to Tetsuo Yamamori, Laboratory for Molecular Analysis of Higher Brain Function, Brain Science Institute, RIKEN, Wako, Saitama 351-0198, Japan. E-mail: tetsuo.yamamori@riken.jp.

K. Ozawa's present address: The Institute of Medical Science Hospital, The Institute of Medical Science, The University of Tokyo, Tokyo 108-8639, Japan. DOI:http://dx.doi.org/10.1523/ENEURO.0019-15.2015

Copyright $\odot 2015$ Sadakane et al.

This is an open-access article distributed under the terms of the Creative Commons Attribution 4.0 International, which permits unrestricted use, distribution and reproduction in any medium provided that the original work is properly attributed. keys by in vivo imaging using virus expression systems (Stettler et al., 2006; Yamahachi et al., 2009), but their observation and analysis focused not on dendritic spines but on axonal structures. Using virus vector-based methods for the labeling of neurons to study their morphology is difficult because the high density of labeled neurons around the injection site also makes the background signal intensity high, thus making such methods unsuitable for the observation of the morphology of fine structures such as dendritic spines. Although dendritic spines in the primate cortex have been extensively analyzed in fixed samples by dye injection methods (Elston et al., 1999; Oga et al., 2013), there has been no report in which dendritic spines were imaged and analyzed in vivo in primate brains.

In this report, we present a method of in vivo imaging of dendritic spines in the marmoset neocortex. The marmoset was chosen as a model animal in our study because the flat surface of a marmoset brain is advantageous for studying the entire cortical region. We mainly had to overcome two technical problems in the in vivo visualization of dendritic spines in the marmoset neocortex. We needed a stronger expression of fluorescent protein because the marmoset brain is more opaque than the brains of smaller animals such as mice. In addition, we needed neurons to be sparsely labeled, because the dense expression obtained by the virus vector method usually causes a high background signal intensity. We, therefore, used an adeno-associated virus (AAV) expression system for the sparse and strong expression of the fluorescent protein in cortical neurons of marmosets, and by twophoton microscopy we were able to observe more clearly dendritic spines labeled by fluorescent proteins.

\section{Materials and Methods}

\section{Animals}

We used six marmosets (all males; body weight, 310420 g; age, 13-22 months); five animals for in vivo imaging and one animal for dye injection. All the protocols used in this study were approved by the Institutional Animal Care and Use Committee of the National Institutes of Natural Sciences, Japan. The experiment was also conducted in accordance with the Guide for the Care and Use of Laboratory Animals of the U.S. National Institutes of Health.

\section{Plasmid construction and AAV preparation}

The constructs used in this study are schematically shown in Figure $1 A$. The Thy $1 \mathrm{~S}$ promoter was cloned from pThy1S-GFP for the sparse labeling of cortical neurons as previously reported (Ako et al., 2011). Owing to capacity limitations of the AAV vector, we truncated $\sim 1.3 \mathrm{~kb}$ of the 5' region of the Thy1S promoter, which is reported to be nonessential for the activity of the promoter (Vidal et al., 1990; Caroni, 1997). The plasmid AAV (pAAV):Thy1Stetracyline transactivator (tTA) was constructed by subcloning the DNA fragments containing the truncated Thy1S promoter and tTA in pAAV-MCS (Agilent Technologies). The pAAV:tetracycline-responsive element (TRE)humanized renilla GFP (hrGFP)-humanized renilla GFP (hrGFP) was constructed by replacing the TurboRed flu- 


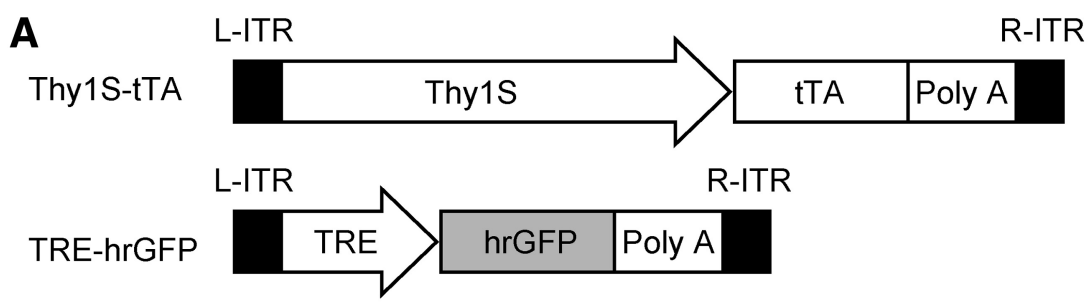

B
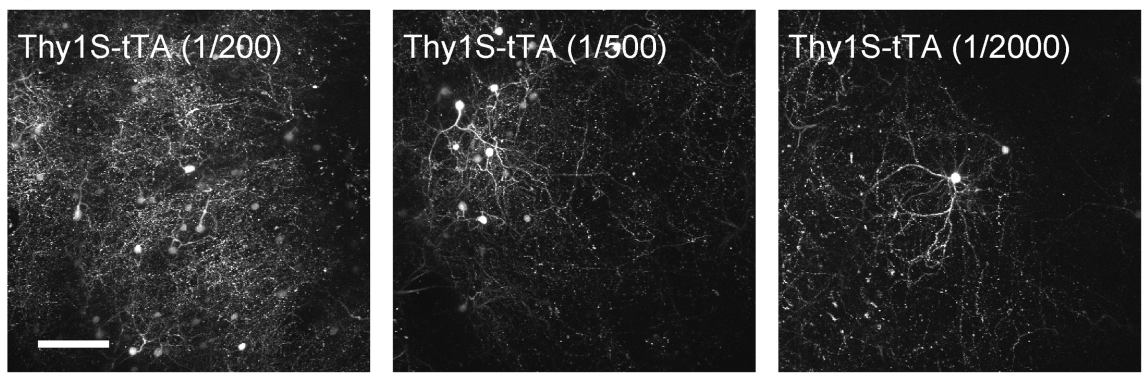

Figure 1. Thy1S promoter drives sparse expression of hrGFP in marmoset cortex. $\boldsymbol{A}$, Schematic illustration of virus constructs. $\boldsymbol{B}$, The expression of hrGFP at different concentrations of virus injection was imaged in fixed brain samples using two-photon microscopy. Left, Low concentration; middle, medium concentration; right, high concentration. Note the difference in labeled cell density at different concentrations. Maximum intensity projections of 71,71 , and 51 slices, respectively, for left, middle, and right panels at intervals of $5 \mu \mathrm{m}$. Scale bar, $100 \mu \mathrm{m}$.

orescent protein (tRFP) sequence of AAV:TRE-tRFP (Watakabe et al., 2014) with hrGFP.

The AAV vectors used in this study have capsids of serotype 1 . They were produced in HEK 293 cells using a helper-virus-free system and were purified twice by $\mathrm{CsCl}_{2}$ density gradient centrifugation and titrated by quantitative PCR, as described previously (Konishi et al., 2008). The final preparations obtained were dialyzed against PBS and diluted as described in the Results section. To prevent adhesion of the AAV vector to glass micropipettes, Pluronic-F68 (Sigma-Aldrich) was added to the vector stock at $0.001 \%$.

\section{Virus injection}

The marmosets were treated by intramuscular injections of ketamine (20 mg/kg; Daiichi Sankyo) and xylazine (1 $\mathrm{mg} / \mathrm{kg}$; Bayer Health Care). Under deep anesthesia induced and maintained by isoflurane (1-2\%) inhalation (Abbott Laboratories), the head of the animal was fixed to a stereotaxic apparatus. Pulse rate, $\mathrm{O}_{2}$ saturation $\left(\mathrm{SpO}_{2}\right)$, and rectal temperature were continuously monitored. $A$ small hole was drilled in the skull using a dental drill. To inject the viruses into the cortex, the dura was punctured using the tip of a 27 ga needle, through which a glass pipette was slowly inserted to a depth of $500 \mu \mathrm{m}$ from the cortical surface. Approximately $0.5 \mu \mathrm{l}$ of a viral solution was injected at a rate of $0.1 \mu \mathrm{l} / \mathrm{min}$. For the imaging of the prefrontal cortex (PFC), our injections were targeted anteroposterior $+18.5 \mathrm{~mm}, 2 \mathrm{~mm}$ to the right from the midline. Following the viral injection, the hole was filled with Spongel, an absorbable gelatin sponge (Astellas Pharma Inc.), and the scalp was sutured. Then the animal was returned to a cage and remained there until the imaging sessions started. To prevent infection, ampicillin (40 mg/kg; Meiji Seika Pharma) was administered intra- muscularly. Carprofen ( $5 \mathrm{mg} / \mathrm{kg}$; Pfizer) was administered intramuscularly as an analgesic and an anti-inflammatory agent. Ampicillin and carprofen were administered immediately after surgery and 2 subsequent days. We waited until an adequate level of gene expression was obtained, which took at least 2 weeks.

\section{In vivo imaging}

Before an imaging session, we constructed an imaging window on the head of the animal. The hole in the skull used for virus injection was expanded to a size of $\sim 2 \times 3$ $\mathrm{mm}^{2}$ using a dental drill, and part of the dura above the injection site was deflected and resected, yielding an incision $\sim 1 \mathrm{~mm}$ in diameter (Fig. 2A). A small coverglass $\sim 4 \times 4 \mathrm{~mm}^{2}$ in size was fixed with dental cement on top of the skull, and the space between the coverglass and the cortex was filled with an agarose gel (1.5\% in artificial CSF; type III-A; Sigma-Aldrich) to minimize vibration. A custom-made metal plate with a hole having an $11 \mathrm{~mm}$ inner diameter was glued to the skull (Figs. 2B,C). This plate was used to fix the head of the animal during the imaging sessions. The same antibiotic, analgesic, and anti-inflammatory agents as those used for the virus injections were administered immediately after window construction and on 2 subsequent days. We started the imaging sessions from 1 to $7 \mathrm{~d}$ after the imaging window construction (Fig. 2D).

In vivo two-photon imaging was performed using a FV1000MPE Multiphoton Laser Scanning Microscope (Olympus) and a water immersion objective lens [25×; numerical aperture (NA), 1.05; Olympus]. Two-photon excitation $(920 \mathrm{~nm})$ was provided by a mode-locked Ti: Sapphire laser (MaiTai Deep See, Spectra-Physics). Fluorescence was detected using a multialkali photomultiplier tube (PMT) without any filter in front of the PMT. The 
A

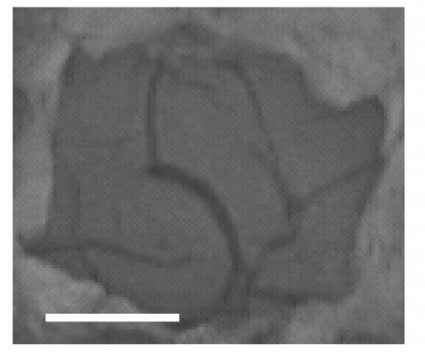

B

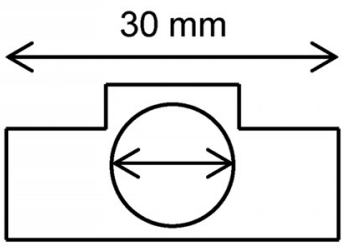

$11 \mathrm{~mm}$

\section{C}

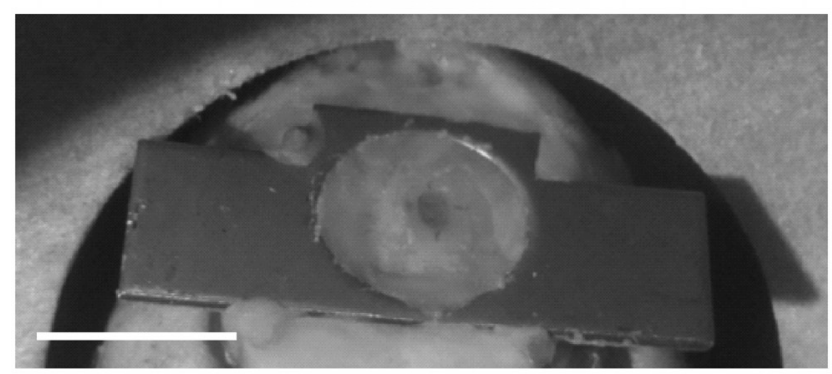

D

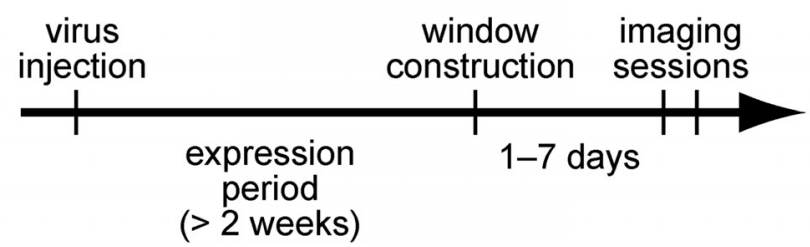

Figure 2. Construction of imaging window. $\boldsymbol{A}$, Craniotomy and durotomy over the target region around the somatosensory cortex. The exposed target region of the marmoset cortex is shown. Scale bar, $500 \mu \mathrm{m}$. B, Illustration of the metal plate used in this study. C, Picture showing the metal plate for fixation, attached to the marmoset head. Scale bar, $10 \mathrm{~mm}$. $\boldsymbol{D}$, Experimental schedule.

regions with fluorescently labeled dendrites were identified without digital zoom (field of view, $508 \times 508 \mu \mathrm{m}^{2}$ ). Then we used an $8 \times$ digital zoom to acquire images of magnified sites including dendritic spines at a resolution of $0.124 \times 0.124 \times 0.2 \mu \mathrm{m}^{3}$ (field of view, $63 \times 63 \mu \mathrm{m}^{2}$ ). These sites were identified the following day, and the images obtained on 2 consecutive days were analyzed. Imaged sites were scattered around a $508 \times 508 \mu \mathrm{m}^{2}$ region and were presumed to contain dendrites originating from different neurons. During imaging sessions, the marmosets were anesthetized with isoflurane (1-2\%). Pulse rate, $\mathrm{SpO}_{2}$, and rectal temperature were continuously monitored.

\section{Dye injection}

A marmoset was sedated with ketamine hydrochloride (25 $\mathrm{mg} / \mathrm{kg}$, i.m.; Daiichi Sankyo) and were overdosed with sodium pentobarbital $(75 \mathrm{mg} / \mathrm{kg}$, i.p.; Dainippon Sumitomo Pharma). The animal was perfused intracardially with $0.1 \mathrm{~m}$ potassium PBS, $\mathrm{pH} 7.2$, followed by $4 \%$ paraformaldehyde (Merck) in $0.1 \mathrm{M}$ PB. A block of tissue was excised from the PFC. Coronal slices of $250 \mu \mathrm{m}$ thickness were prepared from the block. Slices were incubated in DAPI (Sigma-Aldrich) solution to visualize the cell bodies. Pyramidal cells were individually injected with $10 \mathrm{~mm}$ Alexa Fluor 568 (Alexa Fluor 568 Hydrazide in $200 \mathrm{~mm} \mathrm{KCl,}$ A-10441; Thermo Fisher Scientific) under visual guidance with a triple-band fluorescence filter (Semrock). The dyeinjected neurons were imaged using a Leica SP-8 confocal laser scanning microscope and a water-immersion lens (63×; NA, 1.2; Leica) at a resolution of $0.045 \times 0.045$ $\times 0.336 \mu \mathrm{m}^{3}$.

\section{Immunohistochemical analysis}

Marmosets were sedated with ketamine hydrochloride (25 $\mathrm{mg} / \mathrm{kg}$, i.m.; Daiichi Sankyo) and overdosed with sodium pentobarbital $(75 \mathrm{mg} / \mathrm{kg}$, i.p.; Dainippon Sumitomo Pharma). The animals were perfused transcardially with $0.9 \% \mathrm{NaCl}$ and then fixed with $4 \%$ paraformaldehyde in $0.1 \mathrm{~m}$ phosphate buffer, $\mathrm{pH}$ 7.4. Brain samples were cryoprotected with $30 \%$ sucrose $/ 0.1 \mathrm{~m}$ phosphate buffer, $\mathrm{pH}$ 7.0 , and sectioned at thicknesses of $40-50 \mu \mathrm{m}$ using a cryostat. For immunofluorescence analysis, the sections were treated with $80 \%$ methanol/20\% dimethyl sulfoxide solution (Dent's solution) for $>30 \mathrm{~min}$, blocked with $10 \%$ fetal bovine serum, $2 \%$ bovine serum albumin, and $0.5 \%$ Triton X-100 in TBS, pH 7.4, and then incubated overnight with a primary antibody to hrGFP (1:4000; Vitality hrGFP rabbit polyclonal antibody; catalog \#240141, Agilent Technologies) at $4^{\circ} \mathrm{C}$. After incubation with a Cy2-conjugated secondary antibody (anti-rabbit Cy2; 1:1000; Jackson ImmunoResearch), the sections were counterstained with Hoechst 33342 (1:2000; Molecular Probes). For immunostaining with anti-GFAP and Iba1 antibodies, rabbit polyclonal antibodies from Abcam (catalog \#AB7260) and Wako (catalog \#019-19741) were used, respectively, as the primary antibodies, followed by staining with Cy3-conjugated anti-rabbit IgG (1:1000; Jackson ImmunoResearch).

\section{Image analysis}

We used ImageJ (National Institutes of Health), Neurolucida (MBF Bioscience), and custom-made software written on MATLAB (R2009a; MathWorks) for our image analysis. Images were processed with a median filter (2.0 pixel radius) to reduce noise. Dendrites and spines were traced and marked manually in a three-dimensional space. The loss or gain rate of dendritic spines was calculated as the percentage of spines that appeared or disappeared on day 1 , relative to the total number of spines on day 0 . The length of spines was measured from the tip of the spine to the interface with the dendritic stalk (Ji et al., 2010).

\section{Statistical analysis}

All statistical analyses were performed using $R(R$ Development Core Team, 2012). We used the Wilcoxon rank sum test to compare between groups, and we corrected for multiple comparisons when required. Differences were considered to be significant at $p<0.05$. Measurements are reported as the mean and SD. The values of statistical power were calculated using G*Power (http://www.gpower.hhu.de/en.html) and are presented in Table 1. Ex- 
Table 1: Statistical table

\begin{tabular}{llll}
\hline & Data structure & Type of test & Power \\
a & Normality not assumed & Wilcoxon rank sum test & 0.51 \\
b & Normality not assumed & Wilcoxon rank sum test with Holm correction & 1.00 \\
c & Normality not assumed & Wilcoxon rank sum test with Holm correction & 0.97 \\
d & Normality not assumed & Wilcoxon rank sum test with Holm correction & 1.00 \\
e & Normality not assumed & Wilcoxon rank sum test with Holm correction & 0.79 \\
$\mathrm{f}$ & Normality not assumed & Wilcoxon rank sum test with Holm correction & 0.51 \\
$\mathrm{~g}$ & Normality not assumed & Wilcoxon rank sum test with Holm correction & \\
\hline
\end{tabular}

perimental animals were randomly assigned to in vivo or ex vivo conditions.

\section{Results}

\section{Sparse and strong expression system for spine visualization}

To observe the dendritic spines of neurons in the marmoset cortex in vivo, there are the following two requirements: strong and sparse expression of fluorescent protein. The scattering of light in living tissues prevents weak signals from being captured by the detector, particularly in the marmoset brain, which is more opaque than the mouse brain; thus, a strong expression is required. Moreover, to observe fine structures such as spines, the expression of fluorescent protein should be sufficiently strong to adequately label these fine structures. Sparse expression is also required to reduce the intensity of background signals. Even when the expression level of fluorescent protein in each neuron is sufficiently strong, a dense expression of fluorescent protein in neighboring neuronal structures such as dendrites and axons makes the intensity of background signals high, thus preventing the clear observation of dendritic spines.

Our strategy to achieve the requirements described above was to combine the tTA-TRE system and Thy1S promoter (Ako et al., 2011) using two virus vectors. We constructed two AAV vectors: one had the tTA component under the control of the Thy1S promoter (AAV: Thy1S-tTA) and the other had hrGFP under the control of TRE (AAV:TRE-hrGFP). Figure $1 A$ shows a schematic drawing of our virus constructs. Ako et al. (2011) devel-
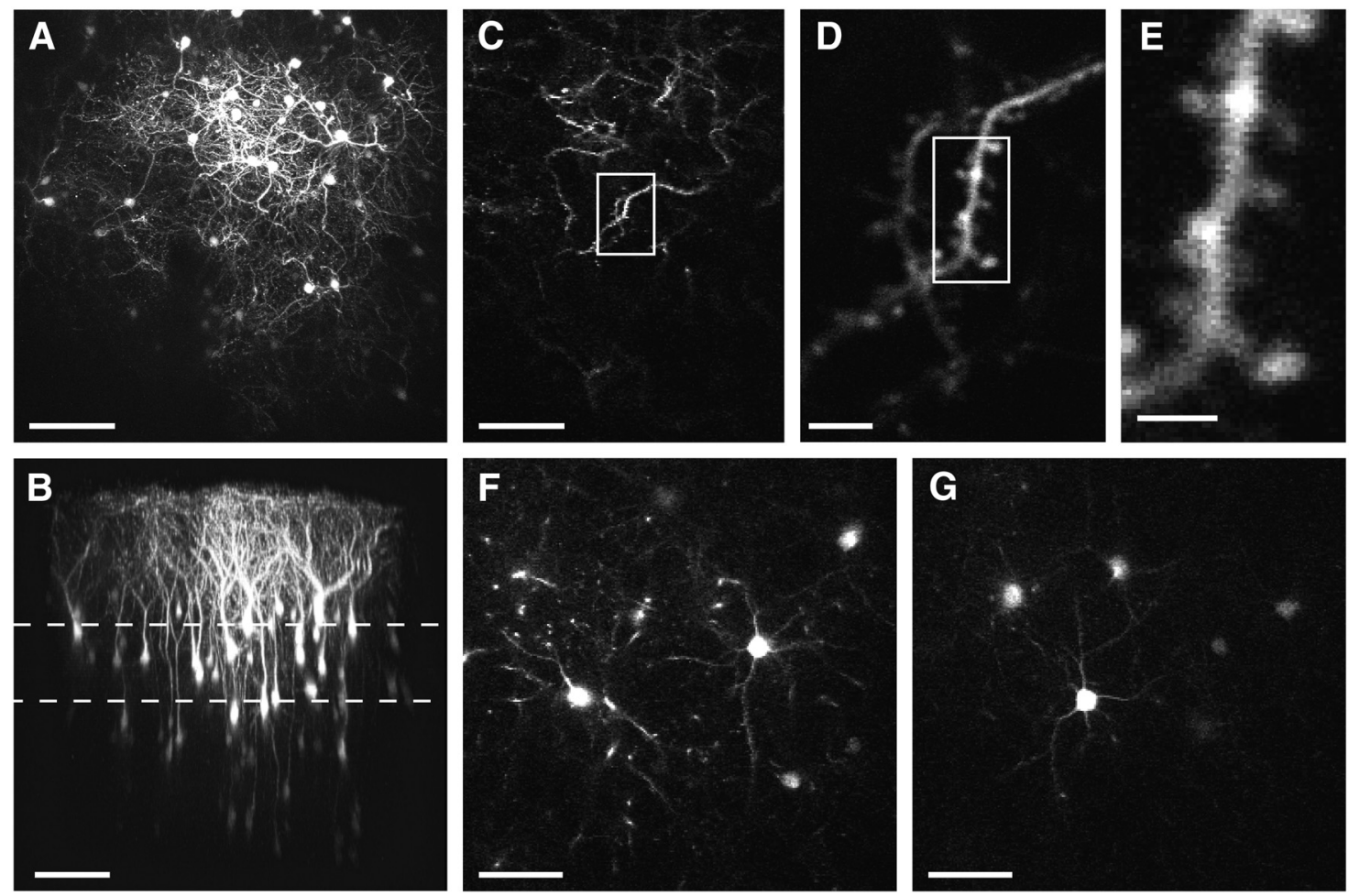

Figure 3. Dendritic spines imaged by in vivo two-photon microscopy. $\boldsymbol{A}$, Maximum intensity projection of the images acquired by in vivo two-photon imaging of marmoset cortex. $B$, Side view of three-dimensional reconstruction of the images of the same site shown in $\boldsymbol{A}$. The depths of the areas shown in $\boldsymbol{F}$ and $\boldsymbol{G}$ are indicated by dashed lines. $\boldsymbol{C}$, Image plane near pial surface. $\boldsymbol{D}$, Magnified image of the boxed area in $\boldsymbol{C}$. $\boldsymbol{E}$, Magnified image of boxed area in $\boldsymbol{D}$ showing dendritic spines. $\boldsymbol{F}$, Image plane at a depth of $220 \mu \mathrm{m}$ showing soma and basal dendrites. G, Image plane at a depth of $330 \mu \mathrm{m}$. Scale bars: $\boldsymbol{A}, \boldsymbol{B}, 100 \mu \mathrm{m} ; \boldsymbol{C}, 50 \mu \mathrm{m}$; $\boldsymbol{D}, 5 \mu \mathrm{m} ; \boldsymbol{E}, 2 \mu \mathrm{m} ; \boldsymbol{F}, \boldsymbol{G}, 50 \mu \mathrm{m}$. 
oped the Thy1S promoter to sparsely label the fine structures of neurons in the mouse neocortex. When expressed by electroporation in the mouse neocortex, the Thy1S promoter drives the gene expression only in a small number of pyramidal neurons in layers $2 / 3$ and 5 . In our expression system, the tTA-TRE system was driven only in Thy1S-positive cells. Hioki et al. (2009) showed that the tTA-TRE system is effective in amplifying transgene expression. The tTA-TRE system has two components, one is tTA and the other is TRE. When tTA binds to the TRE component, the transcription of hrGFP that is under the control of the TRE component is strongly activated.

We injected a mixture of these virus vectors into the marmoset neocortex and first examined the expression in fixed brain samples (Fig. 1B). We were interested in the density of hrGFP-positive neurons and the visibility of each dendrite. In our preliminary mouse experiment, we found that maintaining the concentration of AAV:TREhrGFP at $4.6 \times 10^{12}$ vector genomes $(\mathrm{vg}) / \mathrm{ml}$ and reducing the concentration of AAV:Thy1S-tTA to $\sim 1 / 500$ of the TRE vector $\left(8.8 \times 10^{9} \mathrm{vg} / \mathrm{ml}\right)$ leads to the sparse but strong expression of the fluorescent protein (data not shown). On the basis of this finding, we tested various concentrations of AAV:Thy1S-tTA relative to that of AAV:TRE-hrGFP in marmosets. At an AAV:Thy1S-tTA to AAV:TRE-hrGFP concentration ratio of 1:200, the number of hrGFPpositive neurons was relatively high (Fig. 1B, left). At a concentration ratio of 1:2000, the number of hrGFPpositive neurons was too small (Fig. 1B, right). An AAV: Thy1S-tTA to AAV:TRE-hrGFP concentration ratio of $1: 500$ yielded the most desirable signals in terms of intensity and density (Fig. 1B, middle).

We emphasize the importance of amplifying the expression level of hrGFP using the tTA-TRE system. Simply reducing the titer of the virus leads to a low expression
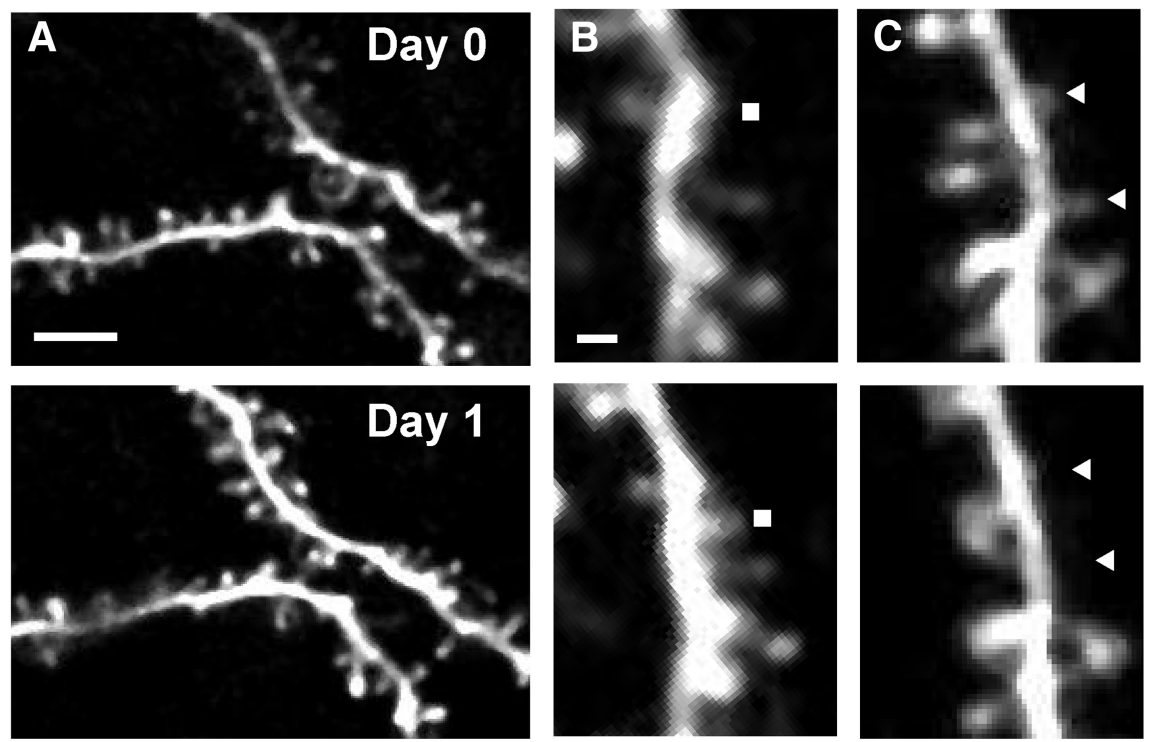

D

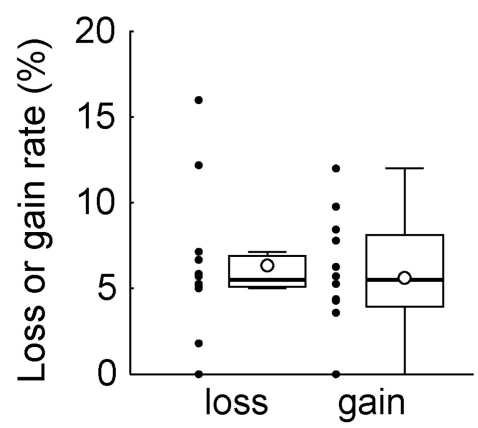

E

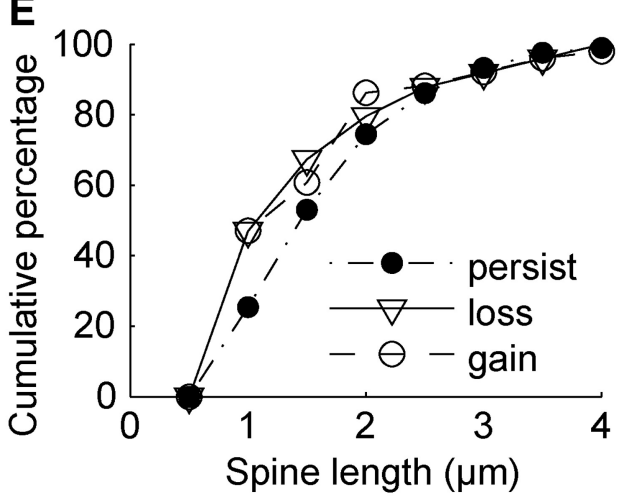

Figure 4. Time-lapse imaging of spines in prefrontal cortex. $\boldsymbol{A}$, The same dendritic regions in the prefrontal cortex were imaged at 24 $\mathrm{h}$ intervals. The top panel shows an image acquired on day 0 ( $7 \mathrm{~d}$ after craniotomy), and the bottom panel shows an image acquired on day 1 . Scale bar, $5 \mu \mathrm{m}$. $\boldsymbol{B}$, The gained spines were identified by manual inspection of two images acquired at $24 \mathrm{~h}$ intervals. A filled rectangle indicates the position of an example of a gained spine. Scale bar, $1 \mu \mathrm{m}$. $\boldsymbol{C}$, The same as $\boldsymbol{B}$ for lost spines. Filled triangles indicate the positions of lost spines. $\boldsymbol{D}$, Box plots showing the spine turnover rate. The open circles in box plots indicate mean values. Black dots indicate values for each site. The whiskers extend to the largest and smallest values within 1.5 times the interquartile range. $\boldsymbol{E}$, Cumulative distributions of spine length in persisting, gained, and lost populations. 
level, thus making it difficult to observe spines in vivo. The combination of titration and amplification was required for the clear visualization of dendritic spines in the marmoset neocortex.

\section{In vivo visualization of dendritic spines in neocortex} To test the feasibility of our viral expression system in vivo, we injected the virus into the marmoset neocortex. After 2 weeks of an expression period, we acquired images from living animals under anesthesia induced by isoflurane. Figure 3 shows in vivo captured images. We were able to visualize each dendritic spine using our virus constructs (Fig. 3C-E). We observed the dendritic spines of apical dendrites located in layer 1 . We were also able to observe the cell bodies located at a depth of $\sim 300 \mu \mathrm{m}$ from the pia (Fig. 3F, at $220 \mu \mathrm{m}$; Fig. 3G, at $330 \mu \mathrm{m}$ ).

We then acquired images repeatedly from the same region over time. Figure 4 shows time-lapse images of the dorsolateral prefrontal cortex, presumably area 8B or 9. The images of the same region of the dendrites were taken over time at $24 \mathrm{~h}$ intervals. During these imaging sessions, the clarity of the imaging window was maintained. Because our samples contained a relatively small number of hrGFP-positive neurons per injected site, we were able to easily identify the same dendrite that we observed in the previous imaging session. The overall shapes of dendrites did not change over this imaging period (Fig. 4A, top and bottom). We marked each spine on the two images (day 0 and day 1 ) by comparing these images side by side, and identified the spines that were "gained" or "lost" during this time interval (Fig. 4B,C). In our experiments, we analyzed 779 spines (12 sites; 34 dendrites; 3 animals; total dendrite length, $2238 \mu \mathrm{m})$; of these spines, 51 were gained (mean across sites, 6.4\%; SD, $4.2 \%$ ) and 49 were lost (mean across sites, 5.6\%; SD, $3.6 \%$; Fig. 4D). The loss or gain rate at the $1 \mathrm{~d}$ interval observed in this study was similar to those in previous studies of layer 5 neurons of the somatosensory cortex of transgenic mice ( $\sim 12 \%$ in $3 \mathrm{~d}$ for both loss and gain; Kim and Nabekura, 2011) and layer $2 / 3$ neurons of ferret $\mathrm{V} 1$ by the virus vector method ( $\sim 4 \%$ in $1 \mathrm{~d}$ for both loss and gain; Yu et al., 2011). We measured spine length by manual tracing using Neurolucida software, and examined the difference between the distribution of the spines that persisted and that of the spines that changed (gained and lost) over the period of imaging. We observed the tendency that the changed spines were shorter than those that persisted (Wilcoxon rank sum test, changed vs persisted, $p=0.0009^{\mathrm{a}}$ ), which means that shorter spines tend to be gained or lost (Fig. 4E).

One of the concerns raised in the two-photon imaging of dendritic spines in mice studies was the activation of glial cells under invasive procedures. Because our methods in the marmoset neocortex include invasive procedures of virus injection and dura opening, we checked the activation of glial cells in our sample of the prefrontal cortex that was used for the in vivo imaging study. We observed the activation of both astrocytes (GFAP; Fig. $5 A-C$ ) and microglias (Iba1; Fig. 5D-F) around the injection site. This observation indicated that experimenters should carefully choose the experimental paradigm when applying the method presented in this article (see Discussion).
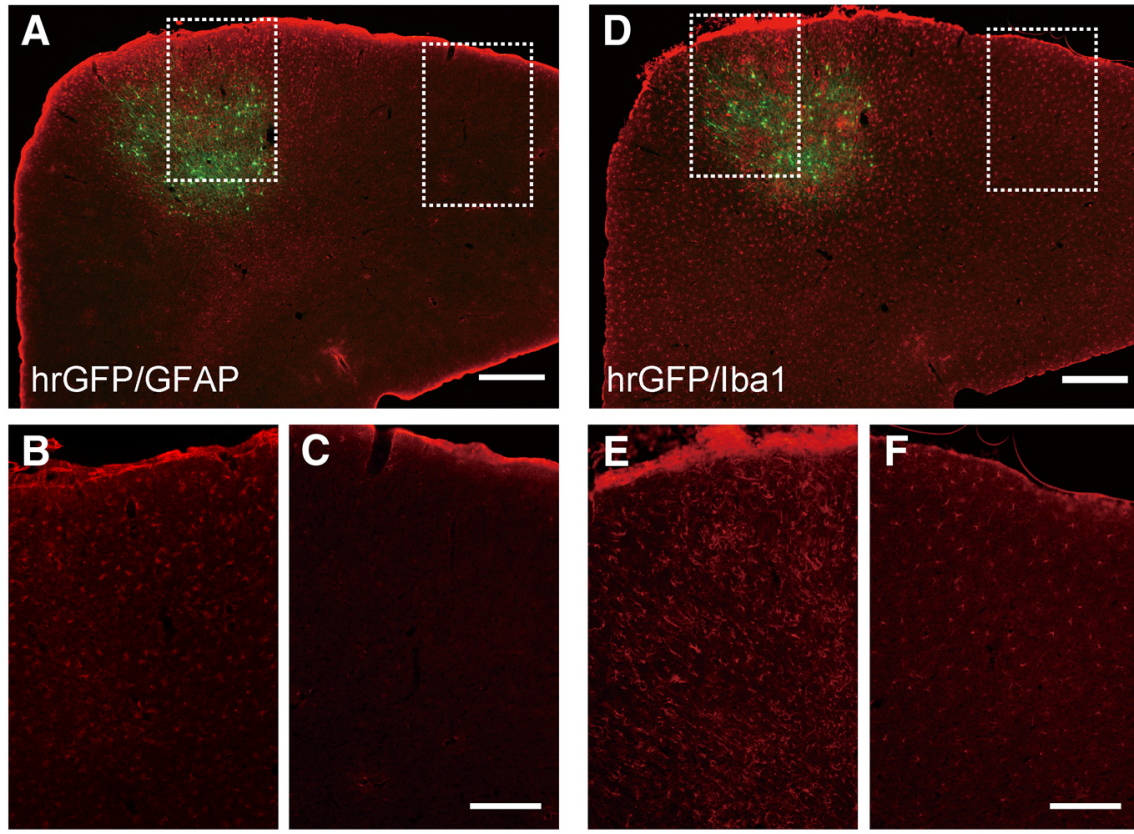

Figure 5. Activation of glial cells. A, Confocal image of a sample immunohistochemically stained with anti-GFAP antibody. Scale bar, $500 \mu \mathrm{m}$. $\boldsymbol{B}$, Magnified image of left boxed area in $\boldsymbol{A}$, near the injection site. $\boldsymbol{C}$, Magnified image of the right boxed area in $\boldsymbol{A}$, distal from the injection site. Scale bar, $200 \mu \mathrm{m}$. $\boldsymbol{D}$, Confocal image of a sample immunohistochemically stained with anti-lba1 antibody. Scale bar, $500 \mu \mathrm{m}$. $\boldsymbol{E}$, Magnified image of the left boxed area in $\boldsymbol{C}$, near the injection site. $\boldsymbol{F}$, Magnified image of the right boxed area in $\boldsymbol{C}$, distal from the injection site. Scale bar, $200 \mu \mathrm{m}$. 

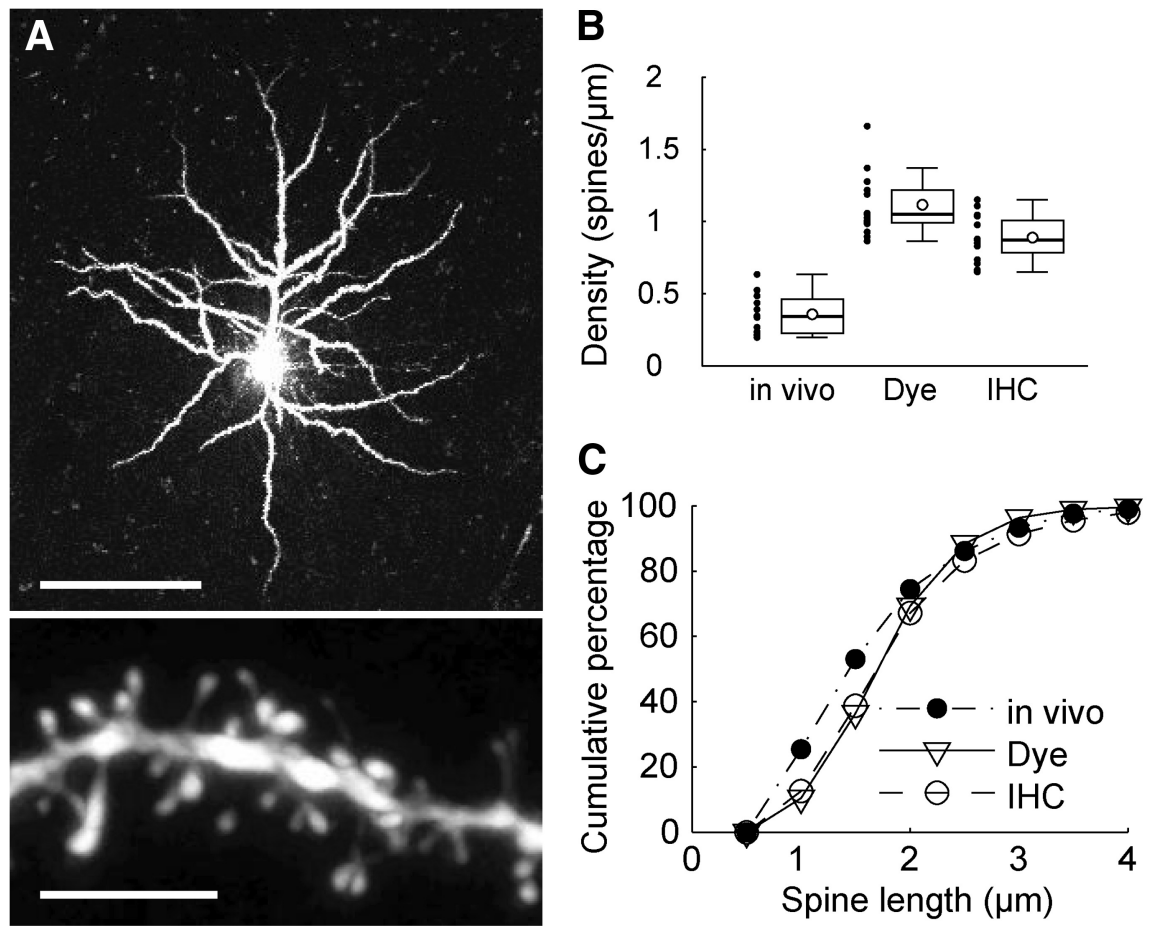

Figure 6. Comparison of dendritic spines between in vivo and ex vivo observations. $\boldsymbol{A}$, Confocal images of ex vivo dye-injected samples. Scale bars: top, $100 \mu \mathrm{m}$; bottom, $5 \mu \mathrm{m}$. B. Box plots showing spine densities in in vivo and ex vivo populations. The open circles in box plots indicate mean values. Black dots indicate values for each site. The whiskers extend to the largest and smallest values within 1.5 times the interquartile range. $\boldsymbol{C}$, Spine densities of in vivo, dye-injected, and IHC samples. $\boldsymbol{D}$, Cumulative distribution of spine length under in vivo and ex vivo conditions.

\section{Comparison between in vivo and fixed samples}

To evaluate our in vivo two-photon microscope images of dendritic spines in the marmoset neocortex, we compared them with those of fixed samples. We wanted to determine whether we observed all existing spines or only a subpopulation of these spines. To clarify this point, we used the method that was used to identify the morphological differences of basal dendrites of pyramidal neurons in different cortical areas from various species, including marmosets and macaques (Elston et al., 1999; Oga et al., 2013). We injected the dye Alexa Fluor 568 into the neurons in the coronal sections of the marmoset prefrontal cortex, corresponding to the region we imaged under the in vivo condition, and observed the spines of apical dendrites of these dye-injected neurons under a confocal microscope (Fig. 6A; one animal; 15 sites; 22 dendrites; 1219 spines; total dendrite length, $1125 \mu \mathrm{m}$ ). Our injection labeled dendritic spines well in the distal region of apical dendrites. We quantified the density and shape of spines in our fixed samples by manual tracing using Neurolucida software, and we compared the results with our in vivo data. The density of spines under our in vivo condition (mean, 0.36 spines/ $\mu \mathrm{m}$; SD, 0.14) was significantly lower than that in dye-stained samples (mean, 1.12 spines $/ \mu \mathrm{m}$; SD, 0.21; Wilcoxon rank sum test with Holm correction, $p=0.00038^{\text {b}}$; Fig. $6 B$ ). The spines observed by in vivo two-photon imaging were shorter than those in dye-stained fixed tissues (Wilcoxon rank sum test with Holm correction, $p=7.6 \times 10^{-12 c}$; Fig. $6 C$ ). This comparison of results between in vivo two-photon imaging and dye staining of fixed samples indicated that the observation of dendritic spines by the in vivo imaging system still has certain limitations.

As for the causes of these limitations, there are two possibilities. The most likely possibility is that because of inefficient visualization, we underestimated the number of spines in in vivo imaging. The second possibility is that the damage caused by AAV injection decreased the number of spines. To determine which of these two possibilities is true, we immunohistochemically stained our samples used in vivo imaging with the antibody to hrGFP immunohistochemically stained [immunohistochemistory $(\mathrm{IHC})$ samples], and then observed these fixed samples by confocal microscopy (two animals; 15 sites; 20 dendrites; 839 spines; total dendrite length, $951 \mu \mathrm{m}$ ). Although one may consider the comparison between the observation in vivo and the same dendritic segments identified in fixed condition, it was difficult for us, in a practical way, to identify the same dendritic segments in the more crowded fixed samples because immunohistochemical staining amplified the signals even in dendrites that express hrGFP weakly. Thus, here we compared the observation in vivo with the dendritic segments at the matching regions from the same samples. The spine density of IHC samples was much higher than that of samples in in vivo imaging (Wilcoxon rank sum test with Holm correction, $p=$ $0.00038^{\text {d; }}$ Fig. $6 B$ ), suggesting that we were not able to detect weaker signals under in vivo two-photon imaging. 
However, the spine density of IHC samples was lower than that of dye-stained fixed samples (Wilcoxon rank sum test with Holm correction, $p=0.0028^{\mathrm{e}}$ ), which also suggests that there may have been some losses of spines owing to tissue damage, as mentioned above. However, the distribution of spine length of $\mathrm{IHC}$ samples largely overlapped with that of dye-stained fixed samples, but not with that of in vivo samples (Fig. 6C). This indicates that the window construction only mildly affected dendritic spines (Wilcoxon rank sum test with Holm correction; IHC vs dye, $p=0.64^{\mathrm{f}}$; IHC vs in vivo, $p=2.2 \times$ $10^{-109}$.

\section{Discussion}

In this study, we established a method of visualizing dendritic spines in the marmoset cortex by in vivo twophoton imaging using a virus expression system. To the best of our knowledge, this is the first demonstration of two-photon imaging of dendritic spines in the neocortex of a living primate.

\section{Merits of this method}

By virus injection, we can control gene expression and monitor the morphology of transduced neurons without affecting other parts of the brain. Even though transgenic marmoset lines in which a specific neuronal population expresses fluorescent protein may be available for in vivo imaging in the near future, virus expression systems have their own merits. One advantage is the shorter time required for their preparation. Generating transgenic primate lines requires a much longer time. Another merit is region-specific manipulation. The use of the combination of transgenic animals and virus expression systems will be beneficial in future studies. For example, a specific cell type can be targeted by the transgenic insertion of an appropriate promoter, and the local expression of a gene may be induced by an appropriate virus expression system.

\section{Technical considerations}

Our method presented in this article still has some limitations. First, there may have been tissue damage due to the procedures used in our study. The dura of the marmoset brain is more opaque than that of the mouse brain, so we dissected the dura to observe dendritic spines in vivo by two-photon imaging. This procedure may be more invasive than that used in mice. In mice, some researchers argued that even removing the skull could perturb the underlying brain tissues and make dendritic spines more unstable (Xu et al., 2007). Indeed, as shown in Figure 5, we observed the activation of glial cells around the injection site. Since a previous study in the primary visual cortex of the ferret (Yu et al., 2011) was conducted under conditions comparable to those in our study (using the Sindbis virus), and examined the activity-dependent morphological plasticity of dendritic spines by comparing active and inactive ocular dominance column, likewise our methods presented in this article may be applicable to studies that compare morphological plasticity in different conditions, such as the level of sensory input or the state of learning. Therefore, we believe that our method is an important technical improvement toward the understanding of the function of the primate neocortex. However, we have to further improve our methods so that the tissue will be less damaged. One possible improvement is to construct an imaging window that permits the longer imaging period, and allows waiting for time until the activity of glial cells subsides. Another improvement could be developing procedures to acquire images through the dura. As part of the process of developing the methods described in this article, we are now on the way to acquire images of axons through the intact dura of the marmoset cortex. In future studies, the improvement of the method for imaging dendritic spines through the dura will lead to less invasive imaging.

The second limitation is in detecting weak signals, as shown in Figure 6. Therefore, we need to amplify such signals or use a detector with high sensitivity.

\section{Importance of examining spines in brains of living primates}

The majority of imaging studies of spines were performed in mice, because many molecular biological techniques are available for mice. Although experiments using mice are important, there are certain functions and structures that only primates have acquired during the course of their evolution. For example, the area specialization of the neocortex is far more evolved in primates than in rodents. In previous studies, the area-specific gene expression profiles in the primate neocortex were examined (Yamamori and Rockland, 2006; Yamamori, 2011; Bernard et al., 2012). Genes selectively expressed in association areas (Komatsu et al., 2005; Takaji et al., 2009; Sasaki et al., 2010) and the primary visual cortex (Takahata et al., 2006; Watakabe et al., 2009) were reported. Importantly, areaselective expressions of these genes are not observed in rodents (Yamamori, 2011). In relation to the gene expression patterns in different cortical areas, previous studies have shown that the density of spines significantly differs among different areas of the primate neocortex (Elston et al., 1999, 2005; Elston and Rockland, 2002). There is a smaller difference in the density of spines among different cortical areas in mice than in primates (Ballesteros-Yáñez et al., 2006). In addition, previous studies by in vivo twophoton imaging of spines in mice showed that there is no significant difference in morphological plasticity among different areas (Zuo et al., 2005). It is, therefore, of great interest to us to determine whether there is a difference in morphological plasticity among different areas in the primate brain, which we are going to investigate using the method described in this report.

\section{References}

Ako R, Wakimoto M, Ebisu H, Tanno K, Hira R, Kasai H, Matsuzaki M, Kawasaki H (2011) Simultaneous visualization of multiple neuronal properties with single-cell resolution in the living rodent brain. Mol Cell Neurosci 48:246-257. CrossRef Medline

Ballesteros-Yáñez I, Benavides-Piccione R, Elston GN, Yuste R, DeFelipe $J$ (2006) Density and morphology of dendritic spines in mouse neocortex. Neuroscience 138:403-409. CrossRef Medline

Bernard A, Lubbers LS, Tanis KQ, Luo R, Podtelezhnikov AA, Finney EM, McWhorter MM, Serikawa K, Lemon T, Morgan R, Copeland C, Smith K, Cullen V, Davis-Turak J, Lee CK, Sunkin SM, Loboda 
AP, Levine DM, Stone DJ, Hawrylycz MJ, et al. (2012) Transcriptional architecture of the primate neocortex. Neuron 73:10831099. CrossRef Medline

Caroni P (1997) Overexpression of growth-associated proteins in the neurons of adult transgenic mice. J Neurosci Methods 71:3-9. Medline

Denk W, Strickler JH, Webb WW (1990) Two-photon laser scanning fluorescence microscopy. Science 248:73-76. Medline

Denk W, Svoboda K (1997) Photon upmanship: why multiphoton imaging is more than a gimmick. Neuron 18:351-357. Medline

Elston GN, Benavides-Piccione R, DeFelipe J (2005) A study of pyramidal cell structure in the cingulate cortex of the macaque monkey with comparative notes on inferotemporal and primary visual cortex. Cereb Cortex 15:64-73. CrossRef Medline

Elston GN, Rockland KS (2002) The pyramidal cell of the sensorimotor cortex of the macaque monkey: phenotypic variation. Cereb Cortex 12:1071-1078. Medline

Elston GN, Tweedale R, Rosa MGP (1999) Cellular heterogeneity in cerebral cortex: a study of the morphology of pyramidal neurones in visual areas of the marmoset monkey. J Comp Neurol 415:3351. Medline

Feng G, Mellor RH, Bernstein M, Keller-Peck C, Nguyen QT, Wallace M, Nerbonne JM, Lichtman JW, Sanes JR (2000) Imaging neuronal subsets in transgenic mice expressing multiple spectral variants of GFP. Neuron 28:41-51. Medline

Fu M, Yu X, Lu J, Zuo Y (2012) Repetitive motor learning induces coordinated formation of clustered dendritic spines in vivo. Nature 483:92-95. CrossRef Medline

Grutzendler J, Kasthuri N, Gan WB (2002) Long-term dendritic spine stability in the adult cortex. Nature 420:812-816. CrossRef Medline

Hioki H, Kuramoto E, Konno M, Kameda H, Takahashi Y, Nakano T, Nakamura KC, Kaneko T (2009) High-level transgene expression in neurons by lentivirus with Tet-Off system. Neurosci Res 63:149154. CrossRef Medline

Holtmaat AJ, Trachtenberg JT, Wilbrecht L, Shepherd GM, Zhang X, Knott GW, Svoboda K (2005) Transient and persistent dendritic spines in the neocortex in vivo. Neuron 45:279-291. CrossRef Medline

Ji Y, Lu Y, Yang F, Shen W, Tang TT, Feng L, Duan S, Lu B (2010) Acute and gradual increases in BDNF concentration elicit distinct signaling and functions in neurons. Nat Neurosci 13:302-309. CrossRef Medline

Kim SK, Nabekura J (2011) Rapid synaptic remodeling in the adult somatosensory cortex following peripheral nerve injury and its association with neuropathic pain. J Neurosci 31:5477-5482. CrossRef Medline

Komatsu Y, Watakabe A, Hashikawa T, Tochitani S, Yamamori T (2005) Retinol-binding protein gene is highly expressed in higherorder association areas of the primate neocortex. Cereb Cortex 15:96-108. CrossRef Medline

Konishi M, Kawamoto K, Izumikawa M, Kuriyama H, Yamashita T (2008) Gene transfer into guinea pig cochlea using adenoassociated virus vectors. J Gene Med 10:610-618. CrossRef Medline

Niu Y, Yu Y, Bernat A, Yang S, He X, Guo X, Chen D, Chen Y, Ji S, Si W, Lv Y, Tan T, Wei Q, Wang H, Shi L, Guan J, Zhu X, Afanassieff M, Savatier P, Zhang K, et al. (2010) Transgenic rhesus monkeys produced by gene transfer into early-cleavage-stage embryos using a simian immunodeficiency virus-based vector. Proc Natl Acad Sci U S A 107:17663-17667. CrossRef Medline

Oga T, Aoi H, Sasaki T, Fujita I, Ichinohe N (2013) Postnatal development of layer III pyramidal cells in the primary visual, inferior temporal, and prefrontal cortices of the marmoset. Front Neural Circuits 7:31. CrossRef Medline

R Development Core Team (2012) R: a language and environment for statistical computing. Vienna, Austria: R Foundation for Statistical Computing.

Sasaki E, Suemizu H, Shimada A, Hanazawa K, Oiwa R, Kamioka M, Tomioka I, Sotomaru Y, Hirakawa R, Eto T, Shiozawa S, Maeda T, Ito M, Ito R, Kito C, Yagihashi C, Kawai K, Miyoshi H, Tanioka Y, Tamaoki N, et al. (2009) Generation of transgenic non-human primates with germline transmission. Nature 459:523-527. CrossRef Medline

Sasaki T, Komatsu Y, Watakabe A, Sawada K, Yamamori T (2010) Prefrontal-enriched SLIT1 expression in old world monkey cortex established during the postnatal development. Cereb Cortex 20: 2496-2510. CrossRef Medline

Stettler DD, Yamahachi H, Li W, Denk W, Gilbert CD (2006) Axons and synaptic boutons are highly dynamic in adult visual cortex. Neuron 49:877-887. CrossRef Medline

Takahata T, Komatsu Y, Watakabe A, Hashikawa T, Tochitani S, Yamamori T (2006) Activity-dependent expression of occ1 in excitatory neurons is a characteristic feature of the primate visual cortex. Cereb Cortex 16:929-940. CrossRef Medline

Takaji M, Komatsu Y, Watakabe A, Hashikawa T, Yamamori T (2009) Paraneoplastic antigen-like 5 gene (PNMA5) is preferentially expressed in the association areas in a primate specific manner. Cereb Cortex 19:2865-2879. CrossRef Medline

Trachtenberg JT, Chen BE, Knott GW, Feng G, Sanes JR, Welker E, Svoboda K (2002) Long-term in vivo imaging of experiencedependent synaptic plasticity in adult cortex. Nature 420:788-794. CrossRef Medline

Vidal M, Morris R, Grosveld F, Spanopoulou E (1990) Tissue-specific control elements of the Thy-1 gene. EMBO J 9:833-840. Medline Watakabe A, Komatsu Y, Sadakane O, Shimegi S, Takahata T, Higo N, Tochitani S, Hashikawa T, Naito T, Osaki H, Sakamoto H, Okamoto M, Ishikawa A, Hara SI, Akasaki T, Sato H, Yamamori T (2009) Enriched expression of serotonin $1 B$ and 2A receptor genes in macaque visual cortex and their bidirectional modulatory effects on neuronal responses. Cereb Cortex 19:1915-1928. CrossRef

Watakabe A, Takaji M, Kato S, Kobayashi K, Mizukami H, Ozawa K, Ohsawa S, Matsui R, Watanabe D, Yamamori T (2014) Simultaneous visualization of extrinsic and intrinsic axon collaterals in Golgilike detail for mouse corticothalamic and corticocortical cells: a double viral infection method. Front Neural Circuits 8:110. CrossRef Medline

Xu HT, Pan F, Yang G, Gan WB (2007) Choice of cranial window type for in vivo imaging affects dendritic spine turnover in the cortex. Nat Neurosci 10:549-551. CrossRef Medline

Yamahachi H, Marik SA, McManus JN, Denk W, Gilbert CD (2009) Rapid axonal sprouting and pruning accompany functional reorganization in primary visual cortex. Neuron 64:719-729. CrossRef Medline

Yamamori T (2011) Selective gene expression in regions of primate neocortex: implications for cortical specialization. Prog Neurobiol 94:201-222. CrossRef Medline

Yamamori T, Rockland KS (2006) Neocortical areas, layers, connections, and gene expression. Neurosci Res 55:11-27. CrossRef Medline

Yu H, Majewska AK, Sur M (2011) Rapid experience-dependent plasticity of synapse function and structure in ferret visual cortex in vivo. Proc Natl Acad Sci 108:21235-21240. CrossRef Medline

Zuo Y, Lin A, Chang P, Gan WB (2005) Development of long-term dendritic spine stability in diverse regions of cerebral cortex. Neuron 46:181-189. CrossRef Medline 